\title{
Natural Language Analyzed with Al-based Transformers Predict Traditional Well-Being Measures Approaching the Theoretical Upper Limits in Accuracy
}

\author{
Oscar Kjell ${ }^{12 *}$, Sverker Sikström ${ }^{1}$, Katarina Kjell ${ }^{1} \&$ H. Andrew Schwartz ${ }^{2}$ \\ ${ }^{1}$ Lund University, Department of Psychology \\ ${ }^{2}$ Stony Brook University, Department of Computer Science
}

\begin{abstract}
We show that using a recent break-through in artificial intelligence-transformers-, psychological assessments from text-responses can approach theoretical upper limits in accuracy, converging with standard psychological rating scales. Text-responses use people's primary form of communication -natural language- and have been suggested as a more ecologically-valid response format than closed-ended rating scales that dominate social science. However, previous language analysis techniques left a gap between how accurately they converged with standard rating scales and how well ratings scales converge with themselves - a theoretical upper-limit in accuracy. Most recently, AI-based language analysis has gone through a transformation as nearly all of its applications, from Web search to personalized assistants (e.g., Alexa and Siri), have shown unprecedented improvement by using transformers. We evaluate transformers for estimating psychological well-being from questionnaire text- and descriptive word-responses, and find accuracies converging with rating scales that approach the theoretical upper limits (Pearson $r$ $=.85, p<.001, N=608$; in line with most metrics of rating scale reliability). These findings suggest an avenue for modernising the ubiquitous questionnaire and ultimately opening doors to a greater understanding of the human condition.
\end{abstract}

${ }^{\star}$ Correspondence to oscar.kjell@psy.lu.se. 
Words are the natural medium with which a person expresses their state of mind. However, in psychology today, research is dominated by asking participants to express themselves in the form of numeric rating scales where complex states of mind are represented by predefined answers from a rating scale. A typical social and psychological article uses 20 measures on average, approximately $87 \%$ of which are closed-ended numeric rating scales ${ }^{1}$. Although rating scales have contributed to important findings in Psychology and other fields, they come with drawbacks. A person asked to communicate their inner thoughts and emotions in response to a question (e.g., Are you satisfied with your life?), normally provides a descriptive, open-ended answer in words (e.g., I'm very satisfied. Most of my expectations are met...), rather than closed-ended numeric or category-based answers (e.g., 6=Agree).

Language reflects who we are ${ }^{2}$ and how we feel ${ }^{3}$. As such, "language is the most common and reliable way for people to translate their internal thoughts and emotions into a form that others can understand. Words and language, then, are the very stuff of psychology and communication"4. This power of language to reflect psychological aspects of a person, is beginning to be possible to quantify. Recent evaluations show that psychologically meaningful scores can be produced by artificial intelligence (AI)-based language assessments ${ }^{3,5}$. Anywhere a survey is being used, respondents could instead be asked to freely describe their mental state rather than forced to adhere to closed-ended responses 5 .

Thus, as an alternative to rating scales, questionnaire language-based assessments involve measuring mental states with open-ended responses that are quantified and analyzed with techniques from $\mathrm{AI}^{5}$. The questionnaire language-based assessments provide structure in its prompt, while also allowing respondents to freely describe their state of mind. Research shows that asking respondents to answer with descriptive word-responses or free text-responses, can be used to predict corresponding rating scales ${ }^{5}$. This was done in seven studies using answers related to satisfaction with life and harmony in life (Pearson's $r=.72, p<.001$ ). Although this method is promising, some hesitation in the adoption of language-based assessments exists due to an accuracy gap. Language-based assessments' correlations to rating scales have fallen short of the accuracy degree with which rating scales can be trusted (i.e., taking into account their reliability, measurement error) - which can be seen as a theoretical upper-limit of possible alignment to a rating scale.

Recent advances in AI-based text analysis have yielded unprecedented performance gains in many traditional applications of AI such as web search, automatic machine translation or question answering. These advances are attributed to a new machine learning technique referred to as the transformer $^{6}$. Transformers are large, general purpose statistical models that have been shown to capture the meaning of words in their context ${ }^{6,7}$; for example, it understands the difference between "I feel great" and "it was a great personal loss". To date the most widely cited transformerlanguage-model is BERT (Bidirectional Encoder Representations from Transformers) ${ }^{7}$. 
Evaluations over a variety of standard AI-tests including language classification tasks (i.e., not psychological assessments tasks) demonstrated that the model typically resulted in $10 \%$ or greater reductions in error compared to earlier models ${ }^{7}$. Being able to represent the different meanings of a word depending on its context, can enable researchers to better capture the meaning of what a person is trying to express.

Here, we examine whether transformers (i.e., BERT) can close the accuracy gap and bring language-based assessments closer to a theoretical upper-limit of accuracy as compared to rating scales. We further examine different aspects of the language-based assessment method to understand how they may be used to modernize surveys and the way we understand the human condition. In short, the current analyses involve applying numeric representations (called word embeddings) from pre-trained language models to quantify respondents' word- and text-responses; and then training these word embeddings to predict the rating scales using multiple linear regression. The accuracy is measured as the Pearson correlation between predicted and observed scores using cross-validation (described in the Methods section). We predict rating scales for concepts relating to the cognitive component of subjective well-being including satisfaction with life and harmony in life, which enable comparisons with previous state-of-the-art ${ }^{5}$.

\section{Results}

\section{Language-based assessments as accurate as rating scales' reliability}

The reliability measures for the Harmony in life scale included $r=.76$ for the mean of inter-item correlations, and $r=.84$ for the mean of item-total correlations; and its previously demonstrated test-retest reliability range from $r=.71$ to $.77^{5,8}$. For the Satisfaction with life scale the mean of the inter-item correlations was $r=.73$, and the mean of the item-total correlations $r=.82$; and the previously demonstrated test-retest reliabilities ranged from $r=.82$ to $.84^{5,8}$.

In theory, the reliability of a measure represents a maximum correlation one might expect to that specific measure given the noise of the measure ${ }^{9}$. Thus, we take the $.71-.84$ reliability scores for the Harmony in life scale, and the .73-.84 for the Satisfaction with life scale to define an upperlimit of how accurately an alternative measure could expect to converge with these measures, given the noise of the measures. The language-based assessments from all word- and text-responses using contextualised word embeddings predict the rating scale rivaling its reliability measures. Observed and predicted Harmony in life scale scores yield a very strong Pearson correlation of .85 ( $p<.001$; Table 1$)$, which is significantly stronger than the mean of inter-item correlations, stronger than the test-retest reliability measures and in line with the mean of item-total correlations. The

correlation between predicted and observed scores for the Satisfaction with life scale also yields a strong correlation of $r=.80$, which is here significantly stronger than its inter-item correlation, and approximately in line with its test-retest and the mean of item-total correlations. 
Table 1.

Comparing Pearson Correlations based on All Responses Combined and Analyzed with Contextualized

Word Embeddings to the Reliability of the Rating Scales

\begin{tabular}{lcc}
\hline Model & HILS & SW \\
LS
\end{tabular}

\section{Reliability measures}

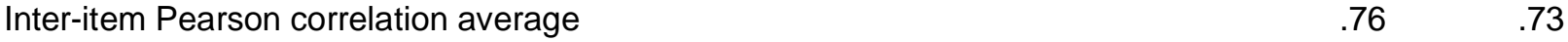

\begin{tabular}{lll} 
Corrected item-total Pearson correlation average & .84 & .82 \\
\hline
\end{tabular}

$\begin{array}{lll}\text { Test-retest reliability } 1^{5} & .71 & .82\end{array}$

Test-retest reliability2 ${ }^{8}$

$.77 \quad .84$

Note. All correlations were significant at $p<.001 . N=608$.

$\mathrm{HIL}=$ Harmony in life; SWL=Satisfaction with life; $\mathrm{S}=$ Scale.

${ }^{\uparrow}=$ significantly higher than Inter-item correlation average; ${ }^{* * *}=p<.001,{ }^{* *}=p<.001$

Current language-based assessments improve previous state-of-the-art

The current language-based assessments for both Harmony in life and Satisfaction with life significantly improve upon the previous state-of-the-art based on a context-free language model and only one word-response format. Using the previous state-of-the-art method for Harmony in life yields a Pearson $r$ of .75 (Table 2); hence our new correlation produces a significant $(p<.001)$ increase of $13 \%$. For Satisfaction with life, the previous method produces an $r$ of .72, where our new method yields a significant $(p<.001)$ increase of $11 \%$ c.f. 5 . 
Table 2.

Comparison of Pearson Correlations Using Contextualized versus Decontextualized Word Embeddings for Individual Word- and Text-Responses

\begin{tabular}{llcccc}
\hline & & \multicolumn{2}{c}{ Text-response } & \multicolumn{2}{c}{ Word-response } \\
& & HIL & SWL & HIL & SWL \\
Word Embeddings & HILS & SWLS & HILS & SWLS \\
\hline Context & BERT & .74 & .74 & .79 & .75 \\
No context & BERT 1 word/docs & $.54 \downarrow$ & $.59 \downarrow$ & .78 & .75 \\
& Latent Semantic Analysis & $.47^{\downarrow}$ & $.46 \downarrow$ & $.75^{\downarrow}$ & .72 \\
\hline
\end{tabular}

Note. All correlations were significant at $p<.001 . N=608$.

$\mathrm{HIL}=$ Harmony in life; SWL=Satisfaction with life; $\mathrm{S}=$ Scale.

Latent Semantic Analysis based on Google 5-gram, 512 dimensions; number of dimensions were optimised as described in ${ }^{5}$ (i.e., based on previous state-of-the-art).

$\downarrow=$ significantly smaller than BERT. See Table SM3 for more comparisons.

\section{Language-based Assessments Can Distinguish Well-Being Dimensions}

To be able to differentiate between concepts, it is important that a measure has noticeably lower correlations with measures from which it theoretically differs. This important characteristic of a psychological measure is called discriminant validity. Even though the Harmony in life scale and the Satisfaction with life scale correlate very strongly $(r=.85)$, both Harmony in life word- and text-responses predict the corresponding rating scale the Harmony in life scale ( $r=.79$ and .74$)$ significantly more accurately than the Satisfaction with life scale ( $r=.66$ and .61; Table. 3; wordresponses: bootstrapped $p=.003$; text-responses: bootstrapped $p=.004)$. But neither Satisfaction with life word- nor text-responses predict the Satisfaction with life scale significantly better than the Harmony in life scale (word-responses: $r=.75$ versus .75, bootstrapped $p=.877$; text-responses: $r=.74$ versus .71 , bootstrapped $p=.284$ ).

Whereas the Harmony in life scale is not significantly more accurately predicted by Harmony in life words as compared with Satisfaction with life words ( $r=.79$ versus $.75, p=.064)$, it is significantly more accurately predicted by Harmony in life text-responses rather than Satisfaction with life text-responses ( $r=.74$ versus .71, $p=.013$ ). And the Satisfaction with life scale is more accurately predicted by the Satisfaction with life word-responses rather than the Harmony in life word-responses $(r=.75$ versus $.66, p<.001)$ and Satisfaction with life text-responses rather than Harmony in life text-responses $(r=.74$ versus $.61, p<.001)$. 
Table 3.

The Construct Specific Validity of Language Models Using Individual Word- and Text-Responses

\begin{tabular}{|c|c|c|c|c|c|}
\hline \multirow{3}{*}{$\begin{array}{l}\text { Language } \\
\text { Model }\end{array}$} & \multirow{3}{*}{ dict } & \multicolumn{4}{|c|}{ Pearson r } \\
\hline & & \multicolumn{2}{|c|}{$\begin{array}{l}\text { Harmony in life } \\
\text { responses }\end{array}$} & \multicolumn{2}{|c|}{$\begin{array}{l}\text { Satisfaction with life } \\
\text { responses }\end{array}$} \\
\hline & & Words & Text & Words & Text \\
\hline \multirow[t]{2}{*}{ BERT } & & $.79^{\uparrow}$ & $.74^{\uparrow}$ & .75 & .71 \\
\hline & LS & .66 & .61 & .75 & .74 \\
\hline \multicolumn{6}{|c|}{$\begin{array}{l}\text { Note. } \\
\text { HIL=Harmony in life; SWL=Satisfaction with life } \\
\downarrow=\text { significantly higher than SWLS prediction. }\end{array}$} \\
\hline \multicolumn{6}{|c|}{$\begin{array}{l}\text { Further, even though the predicted Harmony in life scale and the predicted Satisfaction with life } \\
\text { scale scores are very strongly correlated }(r=.96, p<.001 \text {; Table } 4) \text {, it is possible to train the language } \\
\text { models to differentiate between the two with significant accuracy. This is achieved by training the } \\
\text { word embeddings to predict the difference scores of the rating scales (i.e., the normalized Harmony } \\
\text { in life scale-scores minus the normalised Satisfaction with life scale-scores). The highest accuracy } \\
\text { is achieved using all responses, which yield considerable accuracy }(r=.34, p<.001) \text {, especially } \\
\text { considering the strong correlation between the Harmony in life scale and the Satisfaction with life } \\
\text { scale }(r=.85, p<.001) \text {. }\end{array}$} \\
\hline \multicolumn{6}{|c|}{$\begin{array}{l}\text { Table } 4 . \\
\text { The Discriminant Validity of Language Models: Significantly Predicting the Harmony in life } \\
\text { scale Minus the Satisfaction with life scale }\end{array}$} \\
\hline Language Model & Responses & $\begin{array}{l}\text { Pred } \\
\text { corre } \\
\text { Pred }\end{array}$ & $\begin{array}{l}\text { HILS } \\
\text { with } \\
\text { WLS }\end{array}$ & $\begin{array}{r}\text { Accurac } \\
\text { HILS }\end{array}$ & $\begin{array}{l}\text { edicted } \\
\text { WLS }\end{array}$ \\
\hline \multirow{3}{*}{ BERT } & All & \multicolumn{2}{|c|}{.96} & \multicolumn{2}{|c|}{.34} \\
\hline & Words & \multicolumn{2}{|c|}{.97} & \multicolumn{2}{|c|}{.25} \\
\hline & Text & \multicolumn{2}{|c|}{.96} & \multicolumn{2}{|c|}{.27} \\
\hline
\end{tabular}

Note. $N=608$. All correlations (Pearson $r$ ) were significant at $p<.001$.

Accuracy ( $r$ ) of Predicted HILS minus SWLS = predicting the difference score of the normalised HILS minus the normalised SWLS, where normalisation was achieved by respectively subtracting the column mean from each score. 


\section{Contextualised Word Embeddings Best for Text Response Predictions}

Next, we investigate to what extent different aspects of the language-based assessments contribute to its validity. An important research question concerns whether word- or text-response formats are most suitable for capturing mental states. Previous research shows that descriptive words rather than text-responses yield more accurate predictions of rating scales (word-responses: $r=.72$; textresponses: $r=.49)^{5}$. However, those algorithms were unable to capture the word order (i.e., a context-free language model). Here, contextualised BERT embeddings are compared with decontextualized embeddings by employing BERT context-free and a context-free nontransformer model previously used for language-based assessments referred to as Latent Semantic Analysis ${ }^{10}$.

The contextualised word embeddings produce substantial increments for text-responses compared to when the context is removed. Contextualised BERT significantly increases the predictive accuracy of text-responses as compared to both BERT decontextualized and Latent Semantic Analysis embeddings (Table 2). Compared to the previous state-of-the-art (i.e., Latent Semantic Analysis), contextualised word embeddings produce significantly higher accuracy for both the Harmony in life scale ( $r=.74$ versus $.47, p<.001)$ and the Satisfaction with life scale $(r=.74$ versus $.46, p<.001)$.

The contextualized embeddings did not create substantial improvements for word-responses, where there was only a significant difference between predictions of the Harmony in life scale from Harmony in life word-responses based on BERT versus Latent Semantic Analysis (.79 versus $.75, p<.01$; for Satisfaction with life word-responses: .75 versus .72, $p=.056$ ).

\section{Word responses produce somewhat higher accuracy than text responses}

Analyses based on all word- versus all text-responses analyzed with BERT demonstrate that wordresponses produce more accurate predictions for the Harmony in life scale (.83 versus .79, $p=.002$; Table 5), but not for the Satisfaction with life scale $(.77$ versus $.75, p=.540)$. That word-responses overall tend to produce a slightly higher accuracy in some situations is also reflected in the information content that the responses carry. There is more entropy in the word- (6.98) as compared with the text-responses (6.04). This means that word-responses, although containing fewer words, contain greater amount of the mathematical concept of information; in other words, if one was to store these two pieces of data in their most efficient forms, the word-responses would require more bits on the computer (i.e., they would take up more disk space) than the text-responses even though the text-responses were much longer. That the words from the word-responses comprise the highest entropy is consistent with the results showing that they produce slightly higher correlations, since more entropy gives the machine learning algorithms more information to use. 
Using multiple response formats and questions improves accuracy

Two response formats were significantly more accurate than one response format for the following four relevant combinations of comparisons. The tests compared predictions of the Harmony in life scale based on: Harmony in life word- and text-responses versus only Harmony in life wordresponses $(r=.82$ versus $.79, p<.001)$ and only Harmony in life text-responses $(r=.82$ versus .74 , $p<.001$ ); as well as predictions of the Satisfaction with life scale scores based on: Satisfaction with life word- and text-responses versus only Satisfaction with life word-responses ( $r=.80$ versus .75 , $p<.001)$ and only Satisfaction with life text-responses ( $r=.80$ versus $.74, p<.001)$.

Next, we examine how accurately multiple word- and/or text-responses from different topics/constructs predict rating scales. Responses from two construct questions produced predictions that were significantly more accurate than predictions from one construct question for all but one comparison. The significance tests included comparing the Harmony in life scale predictions based on: Harmony in life and Satisfaction with life word-responses versus only Harmony in life word-responses ( $r=.83$ versus .79, $p<.001)$; and Harmony in life and Satisfaction with life text-responses versus only Harmony in life text-responses ( $r=.79$ versus .74, $p<.001$ ). And comparing the SLWS predictions based on Harmony in life and Satisfaction with life wordresponses versus only Satisfaction with life word-responses ( $r=.77$ versus $r=.75, p=.039$ ); and Harmony in life and Satisfaction with life text-responses versus Satisfaction with life textresponses $(r=.75$ versus $.74, p=.111)$. 
Table 5. Word- versus Text-Responses: Accuracy (r), Entropy, Information Content and Mean (SD) number of words

\begin{tabular}{|c|c|c|c|c|}
\hline Language Response & $\begin{array}{l}\text { r, HILS } \\
\text { prediction }\end{array}$ & $\begin{array}{l}\text { r, SWLS } \\
\text { prediction }\end{array}$ & $\begin{array}{l}\text { Entropy of } \\
\text { Words Input }\end{array}$ & $\begin{array}{l}\text { Mean (SD) of } \mathbf{N} \\
\text { words }\end{array}$ \\
\hline HIL+SWL Words & .83 & .77 & 6.98 & $18.71(1.42)$ \\
\hline HIL+SWL Text & .79 & .75 & 6.05 & $144.0(74.8)$ \\
\hline HIL words + Text & .82 & NA & 6.36 & $78.2(38.4)$ \\
\hline SWL words + Text & NA & .80 & 6.31 & $84.5(46.0)$ \\
\hline HIL Words & .79 & .66 & 6.69 & $9.8(1.0)$ \\
\hline HIL Text & .74 & .61 & 5.94 & $69.4(38.4)$ \\
\hline SWL Words & .75 & .75 & 6.48 & $9.9(0.73)$ \\
\hline SWL Text & .71 & .74 & 5.94 & $75.6(45.9)$ \\
\hline
\end{tabular}

Note. All correlations were significant at $p<.001 . N=608$. BERT large using the second last layer (L23). $\mathrm{HIL}=$ Harmony in life; SWL = Satisfaction with life; $\mathrm{S}=$ Scale.

Word level entropy = Shannon Entropy; Entropy of embedding level = Differential Shannon Entropy.

\section{Discussion}

\section{Beyond State-of-the-Art and the Reliabilities}

Language-based assessments analyzed with modern transformer language models that enable contextualised word embeddings yield unprecedented high predictive accuracy of rating scales. Combining responses from both word- and text-responses about Harmony in life and Satisfaction with life yields the highest accuracy, which is significantly higher than previous methods. The predictive accuracy for the Harmony in life scale is even higher than the rating scales' reliability as it is typically measured and seen as the theoretically highest limit.

These results demonstrate that word- and text-responses contain valuable information in relation to previously validated rating scales, which further emphasises the significance of evidence supporting language-based assessments. This includes evidence showing that they exhibit higher, or competitive, degrees of validity and reliability when compared with rating scales ${ }^{5}$. This has, for example, been shown when comparing language-based assessments' and rating scales' ability to accurately categorise external stimuli of pictures depicting facial expressions including sad, happy and contemptuous. Another study revealed a significant positive correlation between theoretically 
relevant cooperative behaviour and the language-based assessments of harmony in life (Pearson's $r=.18$; and $r=.35$ in participants categorised as prosocials), but not the corresponding rating scale ${ }^{11}$.

\section{Ability to Distinguish Well-Being Dimensions}

The high significant predictive accuracies support the validity of both the rating scales and the language-based assessments. Since the word- and text-responses were presented before the rating scales, the items composing the rating scale did not influence respondents' view of the targeted psychological construct. Interestingly, the language response for a specific construct tended to predict its corresponding rating scale the best. The Harmony in life responses predict the Harmony in life scale better than the Satisfaction with life scale; however, this was not true for the Satisfaction with life responses and the Satisfaction with life scale. Further, whereas the Satisfaction with life scale is better predicted by Satisfaction with life responses than Harmony in life responses; the Harmony in life scale is only better predicted by the HIL text-responses rather than the Satisfaction with life text-responses.

Lastly, despite the very strong correlation between the rating scales, it is possible to create a discriminant model that significantly predicts their difference scores. These findings demonstrate that respondents perceive the constructs differently, and they are able to describe this with both language and through rating scales. The AI methods demonstrate that individuals show surprising concordance between self-reported rating scales and open-ended questions.

\section{Contextualized word embeddings}

Contextualized, as compared to decontextualized, word embeddings particularly increase the predictive accuracy from text-responses. They substantially increase the predictability of textresponses, which almost reaches the same accuracy as descriptive word-responses (only wordresponses for Harmony in life are significantly more predictive than the text-responses, and also with a small effect size). This is an important finding because it opens up the opportunity to make better use of the text-response format.

\section{Complementary Response Formats}

Comparing the strongest correlation when only using one response ( $r=.79$, Table 4$)$ versus using all responses $(r=.85$, Table 3$)$, demonstrates that adding responses increases the predictive accuracy. The different response formats complement each other in both predictive ability and practical advantages. Descriptive words are less demanding to write (i.e., fewer words to write), text is more natural (i.e., less constrained). Further, changing response formats may promote thinking about the same question in different ways, from different perspectives.

\section{Comparisons with Predictions of Subjective States from Social Media Text}

Language-based assessments based on direct prompts/questions produce considerably stronger correlations than those derived from individuals' social media profiles. Research shows that it is 
possible to assess individuals' subjective states of mind by analysing their social media text (e.g., from Facebook and Twitter). These analyses have been demonstrated to rigorously predict psychological and health related outcomes such as satisfaction with life $(r=.57)^{12}$, and personality $(r=.31-.42)^{2}$. But language-based assessments based on prompts are different from social media text analyses, as they resemble rating scales in directly asking individuals to communicate their state of mind rather than using naturally occurring data, which potentially explains the current unprecedented high predictive accuracy.

\section{Limitations, Conclusions and Future Research}

This study focuses on examining the relationships between language-based assessments and rating scales; but it does not compare which of the two are the most valid or reliable. Even though the rating scales have been validated, and used in a wide range of research settings ${ }^{13,14}$, self-reported measures are not objective truth, and future research should compare rating scale and languagebased assessments in predicting theoretically relevant behaviors, biological markers etc. Notably, language-based assessments can also be constructed to predict these outcomes directly, and thus assess mental states independent of rating scales. Lastly, the sample included respondents online from the USA only, generalisation beyond this should be done with caution.

We show that open-ended, text-responses predict rating scales with unprecedented accuracy. The accuracy is not only significantly higher than previous methods, but higher than or rivaling the typical ways that scales' reliability are measured-which is normally conceived as the upper theoretical limit ${ }^{9}$. We also show that using only 10 descriptive words can reveal a lot of psychological information, that contemporary language models produce very accurate predictions from analyzing text-responses, and that combining responses increases the predictive accuracy as compared with only using one response. These results provide promising evidence that language, the most natural way of conveying complex psychological traits and states of mind, can be quantified to improve and modernise current research methods and improve clinical practices. We envision that these methods are applicable for widespread use in scientific research, including fields such as psychology, neuroscience, or medicine. Hence, these findings suggest an avenue for modernising self-report human assessment and ultimately opening doors to a greater understanding of the human condition. 


\section{Participants}

\section{Methods}

The data used here is by convenience pooled from three previously published studies that received ethical approval from the Regional Ethics Board in Lund, and adhered to Swedish laws (Study 3: $N=92$; Study 4: $N=303$; Study 5: $N=296)^{5}$. Participants were recruited using Mechanical Turk (www.mturk.com). Fifty-six participants were excluded for not answering the control items correctly. Out of the remaining 691 participants, an additional 79 were excluded for not reporting USA as nationality; and 4 participants were excluded for not answering all four open-ended questions. The final sample comprised 608 participants from the USA ( 359 females, 249 males, 0 others), with a mean age of $35.0(S D=12.88$, range $=18-74)$ years.

\section{Instruments}

Open-ended questions for harmony in life (i.e., "Overall in your life, are you in harmony or not?") and satisfaction with life (i.e., "Overall in your life, are you satisfied or not?"), were coupled with instructions to either answer with 10 descriptive words or a text-response.

The Harmony in Life Scale $e^{8}$ measures Harmony in life with five items (e.g., "My lifestyle allows me to be in harmony"), coupled with closed-ended rating scales ranging from "1=Strongly Disagree" to "7=Strongly Agree".

The Satisfaction with Life Scale ${ }^{15}$ measures Satisfaction with life with five items (e.g., "I am satisfied with my life") coupled with the same rating scales alternatives as the Harmony in life scale.

For more details of measures see SM.

\section{Procedure}

First, informed consent was obtained informing participants about the general purpose of the studies and their right to withdraw at any time, that their responses were confidential and that they did not involve collecting personal identifiable information. Then, participants were asked to answer the open-ended questions, which were presented in random order. Subsequently, the rating scales were presented in random order. The demographic questions were presented last, followed by debriefing.

The study was carried out in accordance to relevant guidelines and regulations, and approved by the Regional Ethics Committee in Lund (Sweden).

\section{Analytic Method}

The text analyses were carried out in $\operatorname{Text}^{16}$ (version 0.9.11), which is an R-package ${ }^{17}$ specialised in enabling social scientists to use state-of-the-art natural language processing and machine 
learning. In short, the current analyses involve applying word embeddings from pre-trained language models to quantify the word- and text-responses; and then training the word embeddings to predict the rating scales using regression.

Pre-trained word embeddings. To train high-quality word embeddings requires large amounts of text data; and when that is not available it is possible to use a general-purpose language representation model built on other text data. This is known as pre-training. We will compare bidirectional contextual and context-free pre-trained word embeddings. Bidirectional contextual word embeddings are influenced by other words in a text. In "She looked at the bank account", the embedding of "bank" is also influenced by the previous and following context (i.e., "She looked at the ... account"). The primary bidirectional pre-trained model used here comes from Google's open-source model called BERT ("bert-large-uncased”; henceforth BERT; Devlin et al., 2019). BERT represents tokens (c.f. words) with 24 layers comprising 1024 dimensions each. Only the second to last layer was used based on research demonstrating that this layer yields reliable performances for document- and human-level predictions ${ }^{7,18}$. The contextualised embeddings will be compared with context-free embeddings by letting the BERT model only see one word at a time (1 word/document), and using the context-free Latent Semantic Analysis based model from Kjell et al. (2019).

Training word embeddings to rating scales. To examine the relationship between word- and textresponses with rating scales, the word embeddings dimensions of the responses are used as predictors in ridge multiple regression ${ }^{19}$ to predict the rating scale scores. Training is employed using 10-fold cross-validation, where the training set is further split for analysis $(75 \%$ of the training data is used to create models with different penalties) and assessment (25\% used to evaluate the different models). The prediction accuracies are evaluated with Pearson correlation between observed and predicted scores (for more details see SM).

Reliability of the To-Be-Predicted Measure as The Upper Limits of Prediction Accuracies. It is important to consider the upper limits of how accurate rating scales can be predicted. Statistically, an observed correlation between two measures is not only influenced by the relation between the latent traits, but also the reliability of the measures (see the Attenuation Correlation Coefficient ${ }^{9,20}$ ); where the reliabilities of the measures limit the upper bound of the correlational strength that may be found. For example, the measurement of weight and height can be measured with a reliability of near 1.0, which means that a correlation of $r=.4$, represents .4 out of an upper limit of nearly 1.0. In contrast, self-report measures of psychological constructs tend to exhibit considerably lower reliabilities, which thus lowers the possible upper limit. Measures of psychological constructs that are considered well-constructed often display reliabilities around $r=.8$. Hence, an $r=.7$ between actual and predicted scores of such measures can be seen as .7 out of the upper limit of .8. Therefore, we compare correlations between predicted and observed scores with the rating scales' reliability as measured by their test-retest reliabilities (i.e., across time as 
retrieved from previous research), the rating scales' corrected item-total correlations (i.e., the mean of the Pearson correlation between each item and the total score of all other items in the scale) and inter-item correlation average (i.e., the mean of the Pearson correlations among all individual items). We used reliability measures based on Pearson product-moment correlation instead of other reliability metrics, such as Cronbach's alpha and McDonald's omega (which are reported in the supplement material), so that it is directly comparable to the Pearson-correlation of between our predicted scores and the observed scores.

Information Theory. Shannon Entropy ${ }^{21}$ is used to measure how much information a response format comprises. This is a key measure in machine learning as it indicates how much information the algorithms have at their disposal to learn.

Cutoffs. Alpha was set to .05. All correlations were computed as Pearson product-moment correlation coefficients (r). Correlations of .2-.39 are interpreted as weak, .40-.59 as moderate, .6.79 as strong and $.8-1.0$ as very strong.

\section{References}

1. Flake, J. K., Pek, J. \& Hehman, E. Construct Validation in Social and Personality Research: Current Practice and Recommendations. Soc. Psychol. Personal. Sci. (2017) doi:10.1177/1948550617693063.

2. Schwartz, H. A. et al. Personality, gender, and age in the language of social media: The open-vocabulary approach. PloS One 8, e73791 (2013).

3. Eichstaedt, J. C. et al. Facebook language predicts depression in medical records. Proc. Natl. Acad. Sci. 115, 11203-11208 (2018).

4. Tausczik, Y. R. \& Pennebaker, J. W. The psychological meaning of words: LIWC and computerized text analysis methods. J. Lang. Soc. Psychol. 29, 24-54 (2010).

5. Kjell, O. N., Kjell, K., Garcia, D. \& Sikström, S. Semantic measures: Using natural language processing to measure, differentiate, and describe psychological constructs. Psychol. Methods 24, 92 (2019).

6. Vaswani, A. et al. Attention is all you need. in Advances in neural information processing systems 5998-6008 (2017). 
7. Devlin, J., Chang, M.-W., Lee, K. \& Toutanova, K. BERT: Pre-training of Deep Bidirectional Transformers for Language Understanding. in Proceedings of the 2019 Conference of the North American Chapter of the Association for Computational Linguistics: Human Language Technologies, Volume 1 (Long and Short Papers) 4171-4186 (Association for Computational Linguistics, 2019). doi:10.18653/v1/N19-1423.

8. Kjell, O. N. E., Daukantaitè, D., Hefferon, K. \& Sikström, S. The Harmony in Life Scale Complements the Satisfaction with Life Scale: Expanding the Conceptualization of the Cognitive Component of Subjective Well-Being. Soc. Indic. Res. 126, 893-919 (2016).

9. Spearman, C. The proof and measurement of association between two things. Am. J. Psychol. 15, 72-101 (1904).

10. Landauer, T. K. \& Dumais, S. T. A solution to Plato's problem: The latent semantic analysis theory of acquisition, induction, and representation of knowledge. Psychol. Rev. 104, 211240 (1997).

11. Kjell, O., Daukantaitè, D. \& Sikström, S. Computational Language Assessments of Harmony in Life-Not Satisfaction With Life or Rating Scales-Correlate With Cooperative Behaviors. Front. Psychol. 12, (2021).

12. Schwartz, H. A. et al. Predicting individual well-being through the language of social media. in 516-527 (2016).

13. Pavot, W. \& Diener, E. Review of the Satisfaction With Life Scale. in Assessing Well-Being: The Collected Works of Ed Diener (ed. Diener, E.) 101-117 (Springer Netherlands, 2009). doi:10.1007/978-90-481-2354-4_5.

14. Kjell, O. N. \& Diener, E. Abbreviated three-item versions of the satisfaction with life scale and the harmony in life scale yield as strong psychometric properties as the original scales. J. Pers. Assess. 103, 183-194 (2021).

15. Diener, E., Emmons, R. A., Larsen, R. J. \& Griffin, S. The satisfaction with life scale. J. Pers. Assess. 49, 71-75 (1985). 
16. Kjell, O., Schwartz, H. A. \& Giorgi, S. Text: an R-package for analyzing and visualizing human language using natural language processing and deep learning. (2021).

17. R Core Team. R: A Language and Environment for Statistical Computing. (R Foundation for Statistical Computing, 2014).

18. V Ganesan, A., Matero, M., Ravula, A. R., Vu, H. \& Schwartz, H. A. Empirical Evaluation of Pre-trained Transformers for Human-Level NLP: The Role of Sample Size and Dimensionality. Rev. (2021).

19. Hoerl, A. E. \& Kennard, R. W. \%J T. Ridge regression: Biased estimation for nonorthogonal problems. 12, 55-67 (1970).

20. Muchinsky, P. M. The correction for attenuation. Educ. Psychol. Meas. 56, 63-75 (1996).

21. Shannon, C. E. A mathematical theory of communication. Bell Syst. Tech. J. 27, 379-423 (1948).

22. Oppenheimer, D. M., Meyvis, T. \& Davidenko, N. Instructional manipulation checks:

Detecting satisficing to increase statistical power. J. Exp. Soc. Psychol. 45, 867-872 (2009).

23. Sanh, V., Debut, L., Chaumond, J. \& Wolf, T. DistilBERT, a distilled version of BERT: smaller, faster, cheaper and lighter. ArXiv Prepr. ArXiv191001108 (2019).

24. Sikström, S., Kjell, O. N. E. \& Kjell, K. Semantic Excel: An Introduction to a User-Friendly Online Software Application for Statistical Analyses of Text Data. https://osf.io/z9chp (2018) doi:10.31234/osf.io/z9chp.

25. Sikström, S., Kjell, O. N. E. \& Kjell, K. SemanticExcel.com: An Online Software for Statistical Analyses of Text Data Based on Natural Language Processing. in Statistical Semantics: Methods and Applications (eds. Sikström, S. \& Garcia, D.) 87-103 (Springer International Publishing, 2020). doi:10.1007/978-3-030-37250-7_6.

26. Liu, Y. et al. Roberta: A robustly optimized bert pretraining approach. ArXiv Prepr. ArXiv190711692 (2019).

27. Lan, Z. et al. Albert: A lite bert for self-supervised learning of language representations. 
ArXiv Prepr. ArXiv190911942 (2019).

28. Lucas, R. E., Freedman, V. A. \& Cornman, J. C. The short-term stability of life satisfaction judgments. Emot. Wash. DC 18, 1024-1031 (2018).

29. RStudio Team. RStudio: Integrated Development Environment for R. (RStudio, PBC., 2020).

30. Wickham, H. et al. Welcome to the Tidyverse. J. Open Source Softw. 4, 1686 (2019).

31. Hausser, J. \& Strimmer, K. Entropy: estimation of entropy, mutual information and related quantities. R package version 1.2. 1. See Httpstrimmerlab Org (2014).

32. Wickham, H. stringr: Simple, Consistent Wrappers for Common String Operations. (2019).

33. Wickham, H. \& Henry, L. tidyr: Tidy Messy Data. (2020).

34. Jr, F. E. H., Dupont, with contributions from C. \& others, many. Hmisc: Harrell Miscellaneous. (2020).

35. Dowle, M. \& Srinivasan, A. data.table: Extension of 'data.frame'. (2019).

36. Fox, J. \& Weisberg, S. An R companion to applied regression. (Sage publications, 2018).

37. Kuhn, M., Chow, F. \& Wickham, H. rsample: General Resampling Infrastructure. (2020).

38. Revelle, W. psych: Procedures for Psychological, Psychometric, and Personality Research. (Northwestern University, 2019). 


\section{Supplemental Online Materials}

\section{Response Formats and Algorithms}

Answering different response formats or multiple but related construct questions are hypothesised to increase predictive validity compared to answering a single response format or construct question. This hypothesis is not only based on the fact that more information is available, but also on the assumption that each question enables respondents to change perspectives, providing greater opportunities to elaborate on the to-be-measured construct. From a statistical point of view, multiple inputs may also allow the model to conduct paired comparisons of the data, allowing control for some idiosyncratic response patterns of the assessed individual.

\section{Method}

\section{Participants}

Fifty-six participants were excluded for not answering the control items correctly, which were for Study 3: N=13, Study 4, N=24, and Study 5, N=19. Participants' perceived household economic situation ranging from " $1=$ Our income does not cover our needs, there are great difficulties" to "7 = Our income covers our needs, we can save" had a reported mean of $4.44(\mathrm{SD}=1.96)$.

\section{Material}

Below are two complete examples of the instructions for the word- and text-response questions for the harmony in life question ${ }^{5}$.

"Please answer the question by writing 10 descriptive words below that indicate whether you are in harmony or not. Try to weigh the strength and the number of words that describe if you are in harmony or not so that they reflect your overall personal state of harmony. For example, if you are in harmony then write more and stronger words describing this, and if you are not in harmony then write more and stronger words describing that. Write descriptive words relating to those aspects that are most important and meaningful to you. Write only one descriptive word in each box."

"Please answer the question by writing at least a paragraph below that indicates whether you are in harmony or not. Try to weigh the strength and the number of aspects that describe if you are in harmony or not so that they reflect your overall personal state of harmony. For example, if you are in harmony then write more about aspects describing this, and if you are not in harmony then write more about aspects describing that. Write about those aspects that are most important and meaningful to you."

For the questions allowing text-responses, there was a minimum response limit of 100 characters. If a respondent wrote fewer characters the following message was presented: "Please write a little bit more. Please enter at least 100 characters, which is approximately 20 words."

The Harmony in Life Scale ${ }^{8}$ yielded a Cronbach's alpha of .94, and a McDonald's omega hierarchical of .91 in the current dataset. 
The Satisfaction with Life Scale ${ }^{15}$ produced a Cronbach's alpha of .93 and a McDonald's omega hierarchical of .89 in the current dataset.

Control items such as "Answer 'disagree' on this question", where interspersed among the rating scales items; one control item per rating scale. Participants who did not answer all correctly were excluded from the analyses, as this type of attention checks have been demonstrated to increase the quality of datasets ${ }^{22}$.

Demographics included questions concerning age, gender and perceived economic situations. The perceived household economic situations item asked: "Does the total income of your household allow you to cover your needs?", which were coupled with 7 closed-ended alternatives ranging from " 1 = Our income does not cover our needs, there are great difficulties" to "7 = Our income covers our needs, we can save".

\section{Procedure}

The mean time to complete the studies were $14.58(\mathrm{SD}=9.02), 16.44(\mathrm{SD}=19.48)$ and 20.02 $(\mathrm{SD}=10.31)$ minutes for study 3,4 and 5, respectively ${ }^{5}$.

\section{Analytic Method}

Pretrained Word Embeddings. The BERT-large and Latent Semantic Analysis models are also compared with BERT-base ("bert-base-uncased") and DistilBert" ${ }^{23}$ ("distilbert-base-uncased"). These models are based on text retrieved from Wikipedia and a Book corpus. BERT-large comprises 24 layers, where we used layers 23 for the analyses; the BERT-base model comprises 12 layers, where we used layer 11 for the analyses; and DistillBERT has 6 layers, where we used layer 5. For more details about the creation of the BERT model see ${ }^{7}$.

The context-free model provides one word embedding for every word in the pre-trained model. That is, for context-free representations, the word "bank" has the same representation in "bank account" and "river bank". The context-free pre-trained model in this study comes from Semantic Excel (English Space 1) ${ }^{24,25}$; the model has been produced using Latent Semantic Analysis ${ }^{10}$ applied to text from the Google N-gram database (https://books.google.com/ngrams).

To create a word embedding that represents all words in a word-response or all text in a textresponse, the word embeddings of each word are aggregated using the mean of each dimension of the word embedding.

Note that since the advent of transformers and BERT there have been many more models developed such as RoBERTA ${ }^{26}$, ALBERT $^{27}$ as well as multilingual models such as mBERT, which 
can represent more than 100 languages. All these models and more are available to use in $\operatorname{Text}^{16}$.

Training word embeddings to rating scales. The training sets were stratified according to the outcome (y), using 4 bins to stratify over. Further, the search grid for the penalty in ridge regression ranged from $10^{-16}-10^{16}$, with increases of times 10 . For more details see ${ }^{16}$.

Significance testing the prediction accuracy between models. To test the difference between two prediction models of the same outcome, we first compute the error for each prediction (i.e., y- $\hat{y}$ ), and then use a paired sample t-test to compare whether the errors differ between the two models. To test two prediction models of different outcomes (e.g., comparing a model that predicts the Harmony in life scale with one predicting the Satisfaction with life scale) bootstrapping over predictions and observed values was used to create a distribution of correlations for each model. Subsequently, the overlap of the two bootstrapped distributions were compared.

Statistical Cutoffs. Cronbach's alpha and omega were considered good at .70 or above.

\section{Descriptions of the numeric variables}

\section{Results}

Table SM1 shows descriptive statistics of the rating scale variables; which are normally distributed, where all have a skew and kurtosis within \pm 2 . Table SM2 presents the correlations among rating scales, where the Harmony in life scale and the Satisfaction with life scale correlate very strongly (Pearson $r=.85 . p<.001$; see Table SM2)

Table SM1.

Descriptive statistics of numeric variables

\begin{tabular}{llllll}
\hline Variable & Mean & SD & Min-Max & Skew & Kurtosis \\
\hline HILS & 25.18 & 8.03 & $5-35$ & -0.83 & -0.37 \\
SWLS & 22.85 & 8.44 & $5-35$ & -0.58 & -0.72 \\
Age & 35.61 & 12.88 & $18-74$ & 0.81 & -0.40 \\
Economy & 4.44 & 1.96 & $1-7$ & -0.33 & -1.08
\end{tabular}

Notes. HILS = Harmony in life scale; SWLS = Satisfaction with life scale; Economy = Perceived economy. $\mathrm{N}=608$ 
Table SM2.

Pearson correlations among numeric variables
1.
2.
3.

\section{HILS}

\section{SWLS}

$.85^{\star \star \star}$

3. Age

.06

.01

4. Economy

$.47^{\star \star \star}$

$.47^{\star * *}$

$-.03$

Note. HILS = Harmony in life scale; SWLS = Satisfaction with life scale;

Economy $=$ Perceived economy; ${ }^{* * *}=\mathrm{p}<.001 . \mathrm{N}=608$

Language-based Assessments as Accurate as Rating Scale Reliability

Table SM3 shows predictive performance of a variation of language models including Bert base and DistillBERT.

Table SM3. Extended version of Table 2 Including More Types of Word Embeddings.

Comparison of Using Contextualized versus Decontextualized Word Embeddings for Individual Word-and Text-Responses

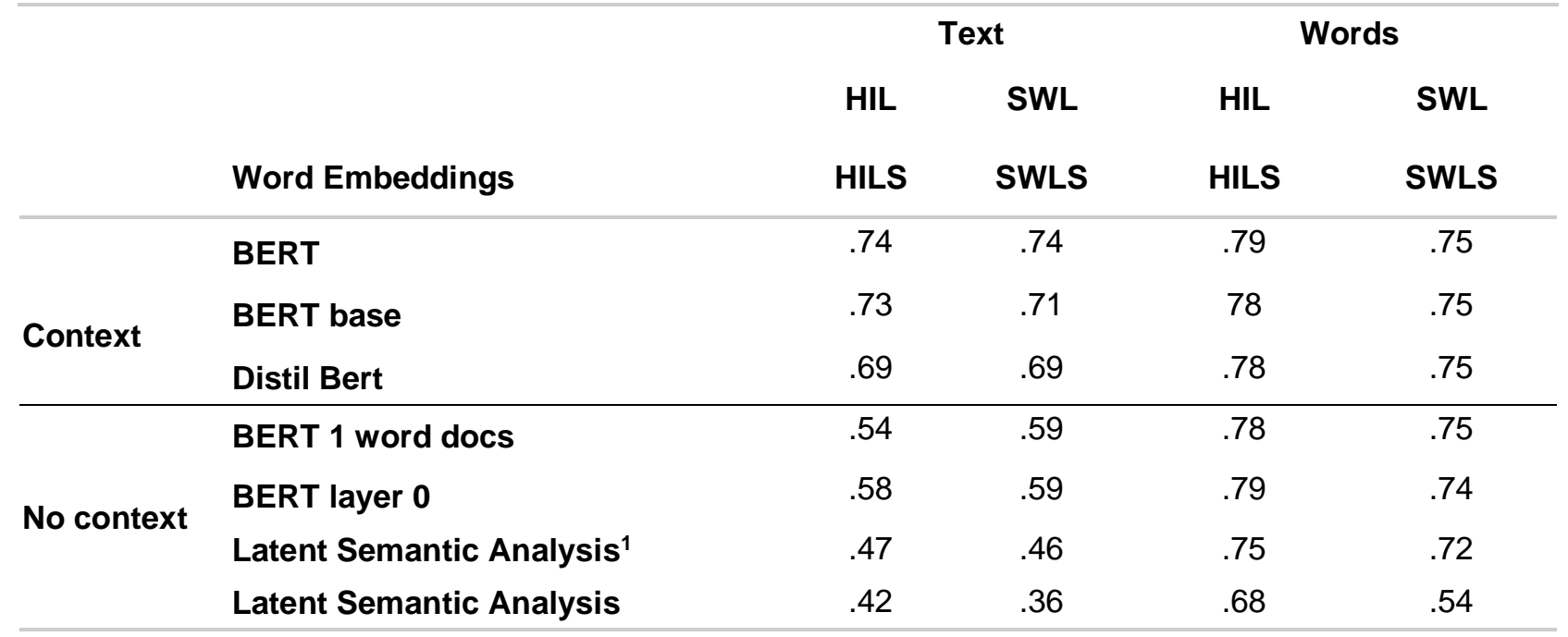

Note. All correlations were significant at $p<.001 . N=608$. BERT using the second last layer (L23). $\mathrm{HIL}=$ Harmony in life; SWL = Satisfaction with life; $\mathrm{S}=$ Scale.

Latent Semantic Analysis based on Google 5-gram, 512 dimensions.

${ }^{1}=$ number of dimensions were optimized as described in ${ }^{5}$ (i.e., based on previous state-of-the-art). 


\section{Discussion}

\section{Limitations of Reliabilities}

The reliability of a scale provides limits on how well they can be predicted. That is, the possible upper limit of the prediction accuracy - how well the assessment captures the intended outcome, is theoretically bounded due to imperfect reliability of the existing to-be-predicted measure. Reliability measures typically used for psychometrics are, however, not always perfect. For example, considering that subjective well-being measures possible changes over time, the testretest reliability is also less than perfect (1.0) due to its valid change over time. Importantly though, the Satisfaction with life scale and the Harmony in life scale measure the individuals' overall/global assessment of their lives, which is known to be stable over time ${ }^{28}$, only changing slowly or due to rare larger events that are less likely to occur over shorter periods of, e.g., around 1-2 months.

Similarly, in the construction of measures for psychological constructs, the aim is often to capture the richness of more complex constructs and to include several items with different wordings to be able to create a total score that can balance out measurement errors. As a result, inter-item and item-total correlation averages should typically not yield a correlation of 1.0. For the purposes of this study, both inter-item and item-total correlation averages can be seen to be especially relevant though. This is because both the Harmony in life scale and the Satisfaction with life scale are relatively short scales, comprising only five items each that are all aimed to capture the most central aspects of the constructs. As a result, this is thus creating relatively high inter-item and item-total correlation averages, and thus provides a considerably high reliability measure.

In the absence of one perfect measure, it is in psychometrics instead common to use several different measures. This is achieved in this study by using the three discussed reliability measures, which may be seen as proxies for the true reliability of the scales.

Further, the current study does not examine the importance for predictability in term of larger number of responses; although research show that the number of descriptive words does not appear to increase the predictability when reaching $9-10$ words ${ }^{5}$; this has not been examined for textresponses (and this particular data set is not appropriate to test this because participants have not written sufficient amounts of text).

\section{R-references}

Analyses were carried out in RStudio ${ }^{29}$, and included using the following packages: tidyverse ${ }^{30}$, entropy ${ }^{31}$, stringr ${ }^{32}$, tidyr ${ }^{33}$, Hmisc ${ }^{34}$, data.table ${ }^{35}$, $\operatorname{car}^{36}$, rsample ${ }^{37}$, and psych ${ }^{38}$. 